\title{
Real-time three-dimensional transesophageal echocardiographic guidance versus fluoroscopic guidance for transvenous temporary cardiac pacemaker implantation during transcatheter aortic valve implantation surgeries
}

\author{
Zhongming Cao ${ }^{1 \#}$, Jindong $\mathrm{Xu}^{2 \#}$, Jian Liu ${ }^{3 \#}$, Min $\mathrm{Wu}^{3}$, Nianjin $\mathrm{Xie}^{4}$, Xiaogang Guo ${ }^{1}$, Huiming Guo ${ }^{3}$, \\ Sheng Wang $^{2 \wedge}$
}

${ }^{1}$ Department of Anesthesiology, Guangdong Provincial Cardiovascular Institute, Guangdong Provincial People's Hospital/Guangdong Academy of Medical Sciences, Guangzhou, China; ${ }^{2}$ Department of Anesthesiology, Guangdong Provincial People's Hospital/Guangdong Academy of Medical Sciences, Guangzhou, China; ${ }^{3}$ Department of Cardiac Surgery, Guangdong Provincial Cardiovascular Institute, Guangdong Provincial People's Hospital/Guangdong Academy of Medical Sciences, Guangzhou, China; ${ }^{4}$ Department of Cardiology, Guangdong Provincial Cardiovascular Institute, Guangdong Provincial People's Hospital/Guangdong Academy of Medical Sciences, Guangzhou, China

Contributions: (I) Conception and design: Z Cao, J Xu, J Liu; (II) Administrative support: S Wang, H Guo; (III) Provision of study materials or patients: J Liang, S Wang, N Xie, X Guo; (IV) Collection and assembly of data: Z Cao, M Wu; (V) Data analysis and interpretation: Z Cao, J Xu; (VI) Manuscript writing: All authors; (VII) Final approval of manuscript: All authors.

\#These authors contributed equally to this work.

Correspondence to: Sheng Wang, MD, PhD. Department of Anesthesiology, Guangdong Provincial People's Hospital/Guangdong Academy of Medical Sciences, Guangzhou, China. Email: shengwang_gz@163.com; Huiming Guo, MD, PhD. Department of Cardiac Surgery, Guangdong Provincial Cardiovascular Institute, Guangdong Provincial People's Hospital/Guangdong Academy of Medical Sciences, Guangzhou, China. Email: guohuiming@163.net.

Background: Fluoroscopic guidance is the traditional method for the implantation of transvenous
temporary cardiac pacemakers (TVTPs). This study aimed to compare the time, effectiveness, and safety
of real-time three-dimensional transesophageal echocardiography (3D TEE) with those of fluoroscopy in
guiding TVTP implantation.

Methods: The records of patients who underwent transcatheter aortic valve implantation (TAVI) guided by real-time 3D TEE or fluoroscopy between July 1, 2016, and June 30, 2020, were retrospectively analyzed. TVTPs were implanted by anesthesiologists via the right internal jugular vein (IJV) in the realtime 3D TEE-guided group (3D TEE group), and by interventional cardiologists via the femoral vein in the fluoroscopy-guided group (fluoro group).

Results: A total of 143 patients (3D TEE-group n=79, and fluoro group $n=64$ ) were included. No statistical differences were observed in the baseline characteristics of the two groups. TVTPs were successfully implanted in all of the patients. The needle-to-pace time was significantly shorter in 3D TEE group than in fluoro group $(5.2 \pm 2.9$ vs. $8.5 \pm 4.6 \mathrm{~min}, \mathrm{P}<0.001)$. Further, the incidence of access complications was significantly lower in $3 \mathrm{D}$ TEE group than in fluoro group $(3.8 \%$ vs. $12.5 \%, \mathrm{P}<0.05)$. One patient in fluoro group who suffered cardiac perforation underwent drainage via pericardiocentesis. No patients in either group died because of TVTP placement. The total complication rates were significantly lower in $3 \mathrm{D}$ TEE group than in fluoro group $(19.0 \%$ vs. $39.1 \%, \mathrm{P}<0.05)$. No statistically significant differences existed between groups in terms of pacing threshold, the incidence of permanent pacemaker insertion after surgery, the length of postoperative intensive care unit (ICU) stay, or the duration of postoperative hospitalization.

\footnotetext{
^ Zhongming Cao, ORCID: 0000-0001-6423-8083; Sheng Wang, ORCID: 0000-0003-0065-0102.
} 
Conclusions: Real-time 3D TEE-guided can be used to effectively, quickly, and safely guide TVTP implantation. The procedure can be performed by properly trained anesthesiologists. Therefore, real-time 3D TEE is a suitable option for guiding perioperative TVTP implantation in patients undergoing cardiac surgery.

Keywords: Transvenous temporary pacing; transesophageal echocardiography (TEE); fluoroscopy; transcatheter aortic valve implantation (TAVI); complications

Submitted Jul 21, 2020. Accepted for publication Sep 25, 2020.

doi: 10.21037/atm-20-5817

View this article at: http://dx.doi.org/10.21037/atm-20-5817

\section{Introduction}

Transvenous temporary cardiac pacemakers (TVTPs) are temporary pacemaker electrode catheters that are inserted into the right ventricle to electrically stimulate the heart. By restoring effective depolarization and cardiac contraction, TVTPs allow an adequate heart rate and cardiac output to be obtained (1). Since being invented in 1952 (2), TVTPs have been widely applied in slow arrhythmia and specific rapid arrhythmia, and $2.7 \%$ of TVTPs are applied as perioperative prophylaxis during cardiac surgery (3).

In recent years, transcatheter aortic valve implantation (TAVI), a revolutionary procedure that is drawing increasing levels of attention worldwide, has gradually replaced median open-heart aortic valve replacement $(4,5)$. TVTPs are needed in TAVI to provide temporary rapid pacing during surgery and to prevent complete atrioventricular block from occurring postoperatively (6). However, this critical technique for TAVI is associated with multiple complications, such as unsafe venous access, incorrect lead placement, sepsis, arterial hematoma, pneumothorax, heart perforation, and life-threatening arrhythmias (3). Fluoroscopic guidance is the standard method for TVTP implantation. In Europe and America, the use of TVTPs appears to be declining $(7,8)$. Nevertheless, with an incidence of serious complications as high as $3.9-5.7 \%(3,9)$, improving the safety of TVTP placement is still a problem worthy of attention.

Furthermore, the use of TVTP is limited by the need to perform fluoroscopy-guided procedures in the catheterization chamber. Ultrasound machines, however, are mobile devices that can be used at the bedside, thus eliminating the need to transport patients. Recent studies to compare ultrasound with fluoroscopy for guiding TVTP implantation reported that ultrasound could successfully guide the placement of TVTPs, significantly shorten the time from placement to pacing, and reduce the rate of TVTPrelated complications (10-13). However, these studies mainly used transthoracic echocardiography (TTE) and had small sample sizes. Therefore, more studies are needed to establish the advantages of ultrasound-guided therapy.

TEE-guided permanent pacemaker implantation in the operating room was described in some early studies $(14,15)$. In recent years, studies have reported that transesophageal echocardiography (TEE) can be used to successfully guide TVTP implantation (16-19). However, the use of TEE for perioperative guidance of TVTP implantation is not recommended by current guidelines (20), and few studies have compared TEE with fluoroscopy for the placement of TVTPs. In many cardiac surgery centers, TEE is already established as a routine monitoring procedure, and the anesthesiologist who performs the procedure is usually qualified with the necessary skills and training. After more than three decades in development, realtime three-dimensional echocardiography (3D TEE) has greatly advanced. It can immediately display intracardial structures and guide the placement of intracardial catheters (21); moreover, 3D TEE may also improve the efficiency and safety of the procedure. With this in mind, we aimed to retrospectively review the data of patients who underwent TVTP implantation guided by real-time 3D TEE or fluoroscopy at our center in order to assess the safety and efficacy of real-time 3D TEE for guiding TVTP implantation. We present the following article in accordance with the STROBE reporting checklist (available at http://dx.doi.org/10.21037/atm-20-5817). 


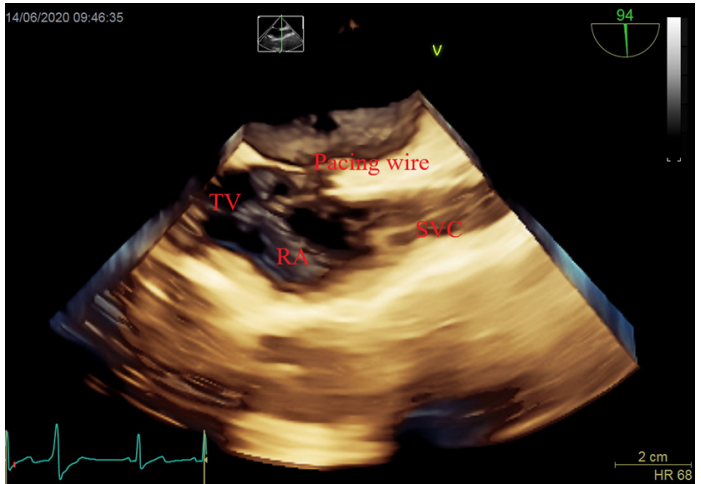

Figure 1 Real-time 3D TEE imaging with the middle esophageal bicaval view was used to guide the tip of the TVTP into the right atrium from the superior vena cava, and then into the right ventricle through the tricuspid valve. 3D TEE, three-dimensional transesophageal echocardiography; TV, tricuspid valve; TVTP, transvenous temporary cardiac pacemaker; RA, right atrium; SVC, superior vena cava.

\section{Methods}

\section{Patients}

The inpatient records of all TAVI patients who received TVTP implantation guided by real-time 3D TEE or fluoroscopy in the Cardiac Surgery Operating Room of Guangdong Provincial People's Hospital between July 1, 2016, and June 30, 2020, were retrospectively reviewed. TVTP implantation in all patients was performed following endotracheal intubation, under general anesthesia in the operating theater. Depending on the preferences of the individual surgeon, some patients underwent TAVI surgery with TVTP implantation using only fluoroscopic guidance, while for others, only real-time 3D TEE was used. This retrospective study was approved by the Institutional Review Committee of Guangdong Provincial People's Hospital (Guangzhou, China) on December 31, 2019 (GDREC2019766H). All procedures performed in this study involving human participants were in accordance with the Declaration of Helsinki (as revised in 2013). Because of the retrospective nature of the research, the requirement for informed consent was waived.

\section{Procedures}

Real-time 3D TEE guidance was performed by two anesthesiologists, who were trained in TEE and TVTP implantation. A Philips iE Elite ultrasound machine (Philips
Medical System, Netherlands) and X7-2T probe, or the GE Vivid E95 system (GE, USA) and 6VT-D probe, were used, along with the corresponding real-time 3D imaging software.

After induction of anesthesia, an esophageal probe was inserted into the esophagus with the aid of a laryngoscope. The neck was sterilized and a sheet was laid, then a sterile sheathed superficial probe was used to guide the puncture of the right internal jugular vein (IJV) in real time. Next, a sheath tube (6F; Edwards, USA) was inserted, sutured, and fixed. Following that, a sterile sheathed bipolar temporary pacemaker catheter (St. Jude Medical, USA) was inserted approximately $10 \mathrm{~cm}$ into the sheath, and the sterile sheet was removed. Subsequently, TEE was used to assess tricuspid regurgitation (TR) and pericardial effusion. Following this, the bicaval view in the middle esophagus (ME) was obtained and transferred into the real-time 3D imaging mode to visualize the lower part of the superior vena cava (SVC) entering into the right atrium, as well as the right atrium, the tricuspid valve (TV), and other structures. The TVTP was slowly delivered by another anesthesiologist until the SVC showed lead imaging (Figure 1). After being inserted into the right atrium, the lead was rotated so that the curved front end was in the direction of the TV, and then inserted so that it passed through the TV into the right ventricle. Subsequently, the four-chamber view in the ME was transferred to the real-time $3 \mathrm{D}$ mode, allowing the $\mathrm{TV}$, the right ventricular inflow tract, and the right ventricular apex to be observed. The pacemaker lead was moved to the right ventricular apex so that the front end of the lead was attached to the endocardium (Figure 2). If the four-chamber view did not clearly show the right ventricular apex, the TEE probe was inserted deep into the stomach to obtain a transgastric longaxis view. Then, in real-time $3 \mathrm{D}$ mode, the pacemaker tip was adjusted to attach to the right ventricular apex (Figure 3).

Finally, a pulse generator (Medtronic Temporary pacemaker 5348, Medtronic, USA) was connected to check whether it could drive cardiac pacing effectively. The sensitivity was set at $2.5 \mathrm{mV}$, and the pacing rate was set at 10 beats/min higher than the patient's heart rate. A pacing threshold of $<10.0 \mathrm{~mA}$ was considered successful.

For the fluoroscopy-guided procedure (Siemens Healthcare GmbH, Germany), the femoral vein approach was adopted according to the standard manner. The procedure was performed by a catheterization team comprising an interventional cardiologist, a nurse, and a technician. The interventional cardiologist observed the pacing leads via digital subtraction angiography (DSA). 


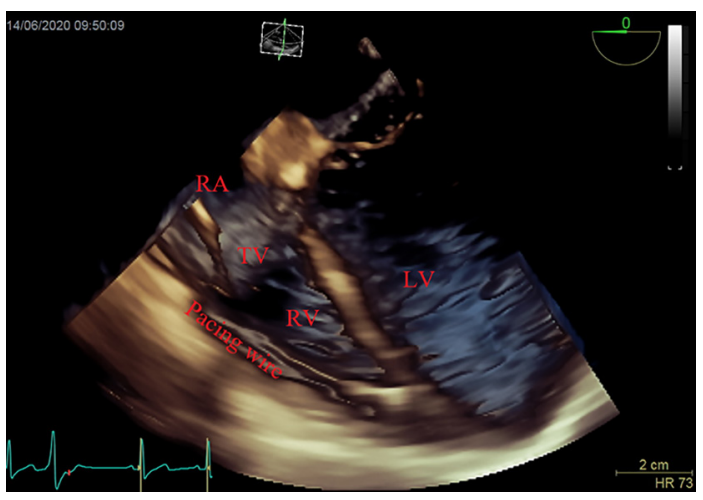

Figure 2 Real-time 3D TEE imaging with the middle esophageal four-chamber view was used to guide the tip of the TVTP into the right ventricle apex via the tricuspid valve. $3 \mathrm{D}$ TEE, threedimensional transesophageal echocardiography; TV, tricuspid valve; TVTP, transvenous temporary cardiac pacemaker; RA, right atrium; RV, right ventricle; $L V$, left ventricle.

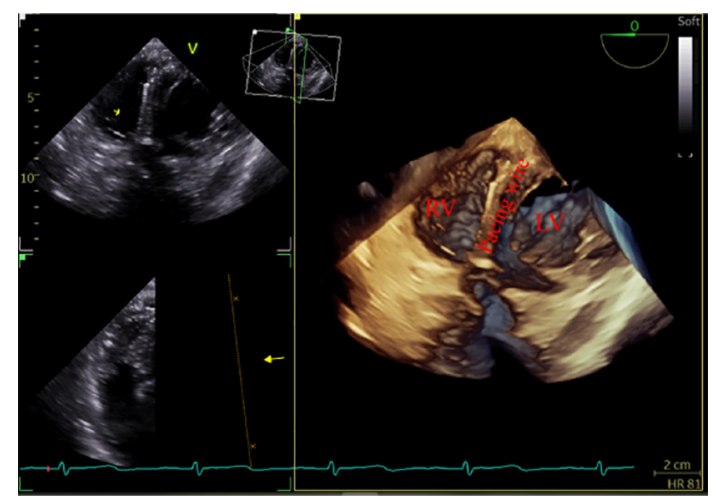

Figure 3 Real-time 3D TEE imaging with the deep transgastric long-axis view was used to guide the tip of the TVTP into the right ventricular apex in a patient with mitral valve replacement. 3D TEE, three-dimensional transesophageal echocardiography; TVTP, transvenous temporary cardiac pacemaker; RV, right ventricle; $L V$, left ventricle

When the tip of the pacing lead was located in the right ventricle and swinging with the heart beat, the electrode was considered to be in contact with the ventricular free wall.

The procedures were video recorded to obtain the operation time and to observe Electrocardiogram (ECG) monitoring. After TVTP implantation, TEE was performed again to assess the TR area and pericardial effusion. When the TR area had increased by $1 \mathrm{~cm}^{2}$ compared to before the operation, this was considered as new or aggravated
TR. The sensor and pacing thresholds for lead placement were recorded. After admission to the intensive care unit (ICU), the threshold for TVTP, venous access infection, and TVTP-related complications were evaluated and recorded by nurses. Serious complications were defined as complications requiring reintervention, including excessive approach bleeding, sepsis, and tamponade.

\section{Statistical analyses}

Data were analyzed using SPSS 21.0 software (IBM, Armonk, NY, USA). Continuous variables were expressed as mean [standard deviation (SD)]. The differences between the two groups were assessed with unpaired $t$-tests. The frequencies of categorical variables were compared using the chi-squared test. $\mathrm{P}<0.05$ was considered to be statistically significant.

\section{Results}

\section{Patient characteristics}

A total of 143 patients were enrolled in this study, including 79 patients in the real-time 3D TEE group (3D TEE group), and 64 patients in the fluoroscopy-guided group (fluoro group). Table 1 lists the patients' preoperative clinical and demographic characteristics. There were no statistically significant differences in baseline characteristics, major diagnosis, access approach, or TR between the two groups.

\section{Procedural characteristics}

Table 2 details the characteristics of the TVTP placement process. TVTPs were successfully implanted in all patients in both groups. The needle-to-pace time in the 3D TEE group was significantly shorter than that in the fluoro group (5.2 \pm 2.9 vs. $8.5 \pm 4.6$ minutes, $\mathrm{P}<0.001)$. However, there were no statistically significant differences in the pacing threshold of TVTP, the need for permanent pacemaker replacement after surgery, the length of postoperative ICU stay, or the duration of postoperative hospitalization between the two groups.

\section{Procedural complications}

Table 3 shows the number and incidence of TVTPrelated complications. The incidence of access-related complications in the 3D TEE group was significantly lower than that in the fluoro group. The incidence of TVTP 
Table 1 Patient demographic characteristics

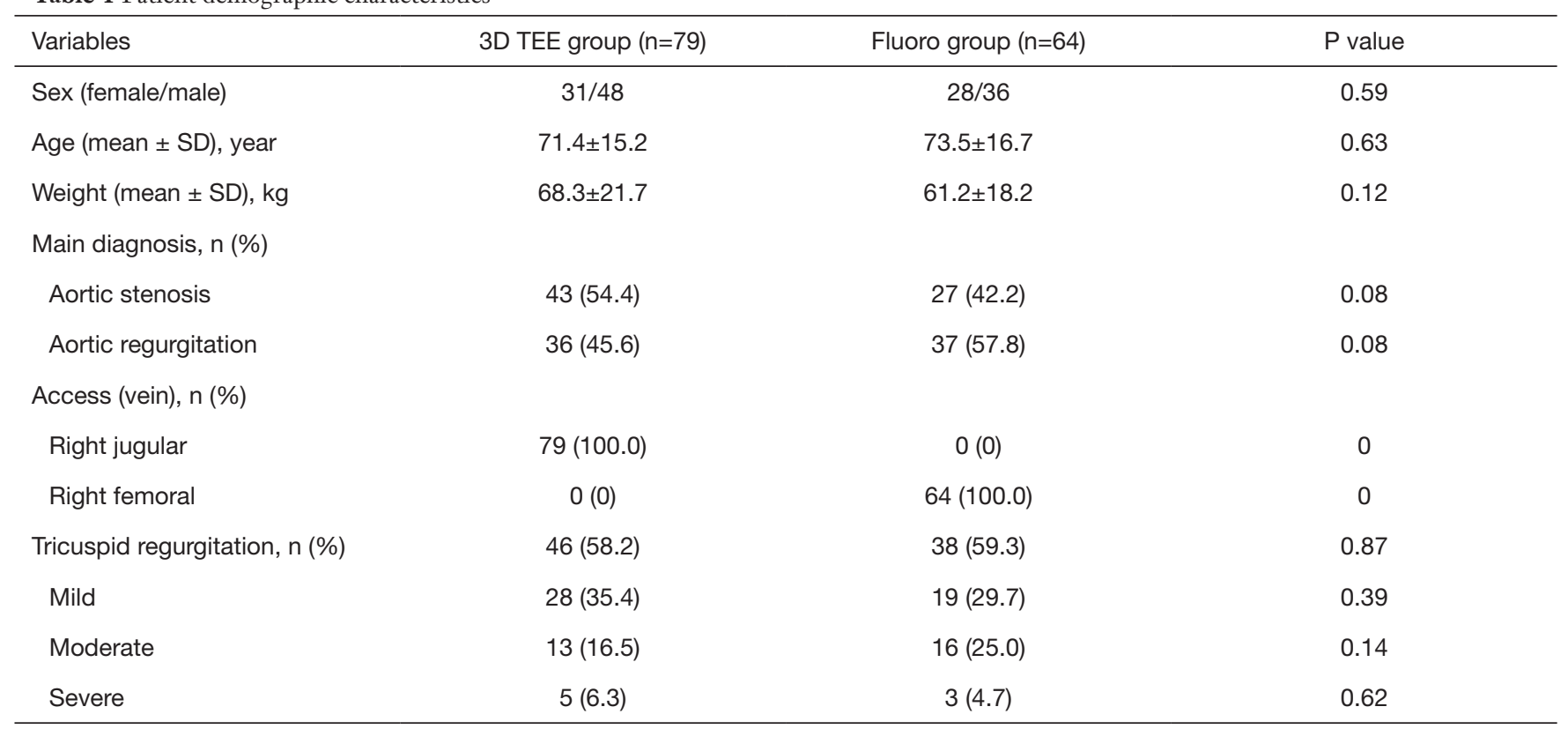

3D TEE-group, real-time 3D TEE-guided group; fluoro group, fluoroscopy-guided group.

Table 2 Procedural characteristics

\begin{tabular}{|c|c|c|c|}
\hline Variables & 3D TEE group $(n=79)$ & Fluoro group $(n=64)$ & $P$ value \\
\hline Procedural success (n, \%) & $79(100.0)$ & $64(100,0)$ & 0 \\
\hline Needle-to-pace time (mean $\pm \mathrm{SD}$ ), min & $5.2 \pm 2.9$ & $8.5 \pm 4.6$ & $<0.001$ \\
\hline Pacing threshold (mean $\pm S D$ ), $m A$ & $5.3 \pm 2.6$ & $6.0 \pm 2.4$ & 0.15 \\
\hline Permanent pacemaker insertion, n (\%) & $6(7.6)$ & $8(12.5)$ & 0.25 \\
\hline $\begin{array}{l}\text { Duration of postoperative hospitalization } \\
\text { (mean } \pm \text { SD), day }\end{array}$ & $4.8 \pm 3.4$ & $5.3 \pm 3.7$ & 0.37 \\
\hline
\end{tabular}

SD, standard deviation.

malfunction requiring repositioning in the 3D TEE group $(5.1 \%)$ was lower than that in the fluoro group (10.9\%), although the difference was not statistically significant. One patient in the fluoro group suffered heart perforation and subsequently underwent drainage via pericardiocentesis. There were no deaths in either group due to TVTP placement. The incidence of complications in the 3D TEE group was significantly lower than that in the fluoro group $(19.0 \%$ vs. $39.1 \%, \mathrm{P}<0.05)$.

\section{Discussion}

This study found that real-time 3D TEE can be used to rapidly, effectively, and safely guide TVTP implantation in TAVI patients under general anesthesia in the operating room. TEE could provide a clear 3D image of the path from the SVC to the right ventricular apex, replacing the classic fluoroscopic approach. To the best of our knowledge, this study is the first to compare real-time 3D TEE with fluoroscopy for guiding TVTP implantation.

Because TAVI needs a central venous catheter to be inserted via IJV puncture for the perioperative administration of drugs, the right IJV approach was chosen for the 3D TEE group. TVTP placement can be performed simultaneously in a sterile area, which simplifies the operation process. A vascular probe was used to guide 
Table 3 Procedural complications

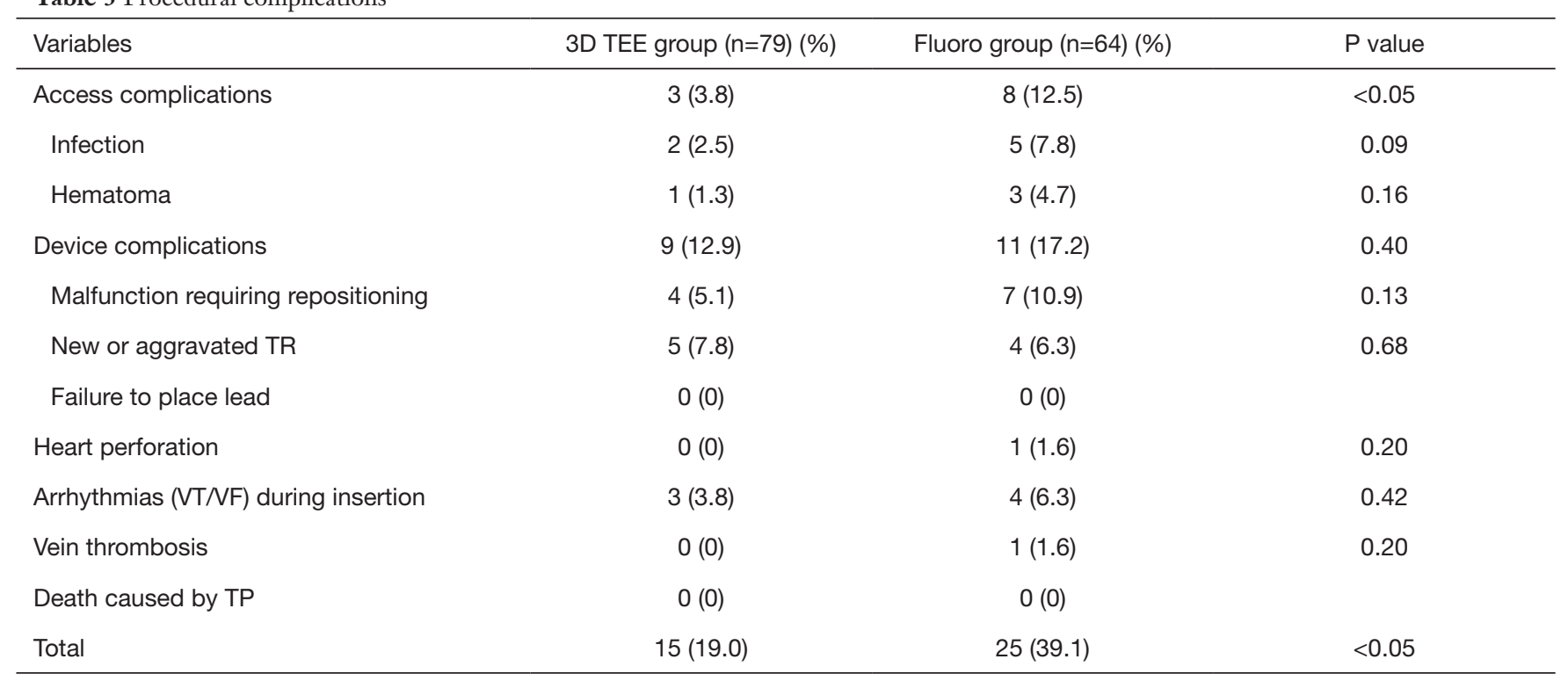

TR, tricuspid regurgitation; VF, ventricular fibrillation; VT, ventricular tachycardia; TP, temporary pacemaker.

the puncture of the IJV, which could reduce access-related complications (22). The path to the right atrium via the IJV is far shorter than that via the femoral vein, and there are fewer branches to enter, giving easier access to the right atrium. Guidelines from the British Cardiac Society also recommend the right internal jugular route, being the most direct route to the right ventricle, as the most suitable approach for inexperienced operators, with a high success rate and fewer complications (23). In our study, upon entering the right atrium through the SVC, the lead was rotated so that the tip of the lead entered directly into the right ventricle without being retracted. However, the femoral vein approach is complicated by the angle between the inferior vena cava and the TV being smaller than that of the SVC and the TV, which makes it more difficult to insert the wire into the tricuspid valve, thus increasing the procedure time. Consistent with the findings of previous studies $(12,23)$, our results showed a higher incidence of lead displacement using the femoral vein approach than using the IJV approach, owing to lower limb activity of the patients. Another advantage of the IJV approach is that the risks of bleeding and infection are lower than with the femoral vein approach (13). Our study made a similar observation, which may be attributable to the insertion of an angiographic catheter into the ipsilateral femoral artery. The puncture of the ipilateral femoral artery carries the risk of bleeding, which also increases the chance of postoperative infection.
With an incidence of $0.5-2.0 \%$ (3), heart perforation is the most serious TVTP-related complication, and it can even prove fatal. Two prospective studies $(12,13)$ have reported that the incidence of heart perforation in the ultrasound-guided group was lower than that in the fluoro group. In our study, emergency pericardiocentesis drainage for heart perforation was also performed on a patient in the fluoro group. This complication may have been caused by the difficulty in clearly determining the level of contact between the tip of the catheter and the myocardium under fluoroscopy. However, under ultrasound, TVTP presented a hyperechoic shadow at the head end, and the endocardium presented medium and low echo shadows. Previous studies have shown that the approach of the pacemaker tip to the right ventricular apex can be observed by 2D TEE (16-19). Our results showed that in most patients, 3D TEE was able to obtain a clear image from the tricuspid valve to the right ventricular apex. Only in patients with mitral valve replacement, mitral annulus calcification, and aortic sinus dilation pressing on the right atrium were the right ventricular septum and apex not clearly shown in the fourchamber view. However, this problem can be easily solved by using the deep transgastric long-axis view. As a result, the process of the pacemaker tip approaching the right ventricular apex could be clearly observed in all patients. Determining the relative positions of the head end and endocardium was easier with real-time $3 \mathrm{D}$ TEE than with fluoroscopy, and meant that the conflict between tip 
orientation and myocardial contraction direction could be avoided.

Because of the high incidence of complications associated with traditional fluoroscopic TVTP implantation, the current guidelines recommend that the use of TVTP be avoided as far as possible (24). However, recent studies to compare ultrasound guidance with fluoroscopic guidance, as well as our study, have shown that ultrasound guidance can effectively reduce the complications associated with TVTP placement. Moreover, real-time 3D TEE can display the relative positions of leads and the intra-cardiac structures more clearly than 2D TEE (25), which simplifies the operation steps, improves the efficiency of implantation, and thus may be promoted and popularized more easily.

In thoracoscopic minimally invasive cardiac surgery, suturing the epicardial pacemaker can prove difficult due to inadequate exposure of the ventricular surface. At this point, using TEE to guide TVTP implantation is a realistic option (26). Moreover, for patients with mechanical ventilation after median sternotomy, and for those in need of emergency temporary pacemaker placement, TEE guidance does not only avoid the risk of transportation to the catheter chamber (27), but it can also avoid the unclear ultrasonic window of TTE (16). Therefore, real-time 3D TEE guidance is a fast and effective treatment approach in the perioperative period of cardiac surgery.

Studies have shown that the outcomes of TVTP implantation by non-cardiovascular physicians are not affected as long as appropriate training is provided (28). At our center, TEE-guided TVTP implantation is performed in the operating room by trained and experienced anesthesiologists. Our findings suggest that the safety and efficacy of TVTP implantation performed by anesthesiologists are no worse than those when the procedure is performed by cardiologists. This is largely due to the fact that 3D TEE allows the intracardial structure and TVTP tip to be visualized, which greatly simplifies the TVTP implantation procedure. Non-cardiologists who master this technique are able to quickly place TVTPs in patients in an emergency.

\section{Limitations}

The present study has both strengths and limitations. Significant strengths of the study were that the baseline characteristics of the groups were similar before surgery and that both groups underwent surgery in the same environment, which increased the comparability of the parameters between the two groups. However, this was a single-center, nonrandomized, small-sample retrospective study, and the procedures were performed by different physicians. In the future, a more convincing conclusion should be pursued in a multicenter, prospective study with a larger sample size.

\section{Conclusions}

In conclusion, our study shows that compared with fluoroscopy, real-time 3D TEE can be used to effectively, quickly, and safely guide TVTP implantation. This procedure can be performed by anesthesiologists with proper training. Therefore, real-time 3D TEE guidance may become the preferred method for perioperative TVTP implantation in cardiac surgery patients.

\section{Acknowledgments}

We thank all our cardiac surgical and cardiology team colleagues for their valuable input.

Funding: This work was supported by a grant from the National Key Research and Development Program (2018YFC2001803).

\section{Footnote}

Reporting Checklist: The authors have completed the STROBE reporting checklist. Available at http://dx.doi. org/10.21037/atm-20-5817

Data Sharing Statement: Available at http://dx.doi. org/10.21037/atm-20-5817

Conflicts of Interest: All authors have completed the ICMJE uniform disclosure form (available at http://dx.doi. org/10.21037/atm-20-5817). The authors have no conflicts of interest to declare.

Ethical Statement: The authors are accountable for all aspects of the work in ensuring that questions related to the accuracy or integrity of any part of the work are appropriately investigated and resolved. This retrospective study was approved by the Institutional Review Committee of Guangdong Provincial People's Hospital (Guangzhou, China) on December 31, 2019 (GDREC2019766H). All procedures performed in this study involving human participants were in accordance with the Declaration of 
Helsinki (as revised in 2013). Because of the retrospective nature of the research, the requirement for informed consent was waived.

Open Access Statement: This is an Open Access article distributed in accordance with the Creative Commons AttributionNonCommercial-NoDerivs 4.0 International License (CC BY-NC-ND 4.0), which permits the non-commercial replication and distribution of the article with the strict proviso that no changes or edits are made and the original work is properly cited (including links to both the formal publication through the relevant DOI and the license). See: https:// creativecommons.org/licenses/by-nc-nd/4.0/.

\section{References}

1. Lopez AJ, Villuendas SR, Garcia GC, et al. (Temporary pacemakers: current use and complications). Rev Esp Cardiol 2004;57:1045-52.

2. Zoll PM. Resuscitation of the heart in ventricular standstill by external electric stimulation. $\mathrm{N}$ Engl J Med 1952;247:768-71.

3. Tjong FVY, de Ruijter UW, Beurskens NEG, et al. A comprehensive scoping review on transvenous temporary pacing therapy. Neth Heart J 2019;27:462-73.

4. Waksman R, Rogers T, Torguson R, et al. Transcatheter aortic valve replacement in low-risk patients with symptomatic severe aortic stenosis. J Am Coll Cardiol 2018;72:2095-105.

5. Garg A, Rao SV, Visveswaran G, et al. Transcatheter Aortic Valve Replacement Versus Surgical Valve Replacement in Low-Intermediate Surgical Risk Patients: A Systematic Review and Meta-Analysis. J Invasive Cardiol 2017;29:209-16.

6. Nishimura RA, Otto CM, Bonow RO, et al. 2017 AHA/ ACC Focused Update of the 2014 AHA/ACC Guideline for the Management of Patients With Valvular Heart Disease: A Report of the American College of Cardiology/American Heart Association Task Force on Clinical Practice Guidelines. J Am Coll Cardiol 2017;70:252-89.

7. Baker RP, Wilson DG. Experiences of Temporary Transvenous Pacing in the South West of England vs the US National Inpatient Sample. Chest 2019;155:1314-5.

8. $\mathrm{Ng} \mathrm{A}$, Lau JK, Chow V, et al. Outcomes of 4838 patients requiring temporary transvenous cardiac pacing: $\mathrm{A}$ statewide cohort study. Int J Cardiol 2018;271:98-104.

9. Metkus TS, Schulman SP, Marine JE, et al. Complications and Outcomes of Temporary Transvenous Pacing: An Analysis of $>360,000$ Patients From the National Inpatient Sample. Chest 2019;155:749-57.

10. Blanco P. Temporary transvenous pacing guided by the combined use of ultrasound and intracavitary electrocardiography: a feasible and safe technique. Ultrasound J 2019;11:8.

11. El Nasasra A, Alnsasra H, Zahger D, et al. Feasibility and safety of exclusive echocardiography-guided intravenous temporary pacemaker implantation. J Echocardiogr 2019;17:157-61.

12. Ferri LA, Farina A, Lenatti L, et al. Emergent transvenous cardiac pacing using ultrasound guidance: a prospective study versus the standard fluoroscopy-guided procedure. Eur Heart J Acute Cardiovasc Care 2016;5:125-9.

13. Pinneri F, Frea S, Najd K, et al. Echocardiographyguided versus fluoroscopy-guided temporary pacing in the emergency setting: an observational study. J Cardiovasc Med (Hagerstown) 2013;14:242-6.

14. Artrip JH, Sukerman D, Dickstein ML, et al. Transesophageal echocardiography guided placement of a coronary sinus pacing lead. Ann Thorac Surg 2002;74:1254-6.

15. Plotkin IM, Collard CD, Aranki SF, et al. Percutaneous coronary sinus cannulation guided by transesophageal echocardiography. Ann Thorac Surg 1998;66:2085-7.

16. Krishnan U, Karthikeyan VJ, Trinh J, et al. First report of the use of transesophageal echocardiography to position a temporary pacing wire. J Cardiothorac Vasc Anesth 2014;28:110-1.

17. Kumar B, Dutta V, Mishra A, et al. Intraoperative transesophageal echocardiography for positioning transvenous temporary pacing wire. J Cardiothorac Vasc Anesth 2015;29:e2-3.

18. Lang RM, Badano LP, Tsang W, et al. EAE/ASE recommendations for image acquisition and display using three-dimensional echocardiography. Eur Heart J Cardiovasc Imaging 2012;13:1-46.

19. Lerner RP, Haaland A, Lin J. Temporary transvenous pacer placement under transesophageal echocardiogram guidance in the Emergency Department. Am J Emerg Med 2020;38:1044.e3-1044.e4.

20. D’Alonzo RC, Rodriquez E, Ryan JW. Percutaneous coronary sinus catheter placement aided by 3 -dimensional transesophageal echocardiography. Anesth Analg 2010;110:722-4; discussion 724.

21. Brass P, Hellmich M, Kolodziej L, et al. Ultrasound guidance versus anatomical landmarks for internal 
jugular vein catheterization. Cochrane Database Syst Rev 2015;1:CD006962.

22. Choice of route for insertion of temporary pacing wires: recommendations of the Medical Practice Committee and Council of the British Cardiac Society. Br Heart J 1993;70:592.

23. Hynes JK, Holmes DJ, Harrison CE. Five-year experience with temporary pacemaker therapy in the coronary care unit. Mayo Clin Proc 1983;58:122-6.

24. Brignole M, Auricchio A, Baron-Esquivias G, et al. 2013 ESC Guidelines on cardiac pacing and cardiac resynchronization therapy: the Task Force on cardiac pacing and resynchronization therapy of the European Society of Cardiology (ESC). Developed in collaboration with the European Heart Rhythm Association (EHRA). Eur Heart J 2013;34:2281-329.

25. Mediratta A, Addetia K, Yamat M, et al. 3D

Cite this article as: Cao Z, Xu J, Liu J, Wu M, Xie N, Guo X, Guo H, Wang S. Real-time three-dimensional transesophageal echocardiographic guidance versus fluoroscopic guidance for transvenous temporary cardiac pacemaker implantation during transcatheter aortic valve implantation surgeries. Ann Transl Med 2020;8(19):1227. doi: 10.21037/atm-20-5817 echocardiographic location of implantable device leads and mechanism of associated tricuspid regurgitation. JACC Cardiovasc Imaging 2014;7:337-47.

26. Lei Q, Wei X, Huang K, et al. Intraoperative implantation of temporary endocardial pacing catheter during thoracoscopic redo tricuspid surgery. Heart Lung Circ 2019;28:1121-6.

27. Knight PH, Maheshwari N, Hussain J, et al. Complications during intrahospital transport of critically ill patients: Focus on risk identification and prevention. Int J Crit Illn Inj Sci 2015;5:256-64.

28. Birkhahn RH, Gaeta TJ, Tloczkowski J, et al. Emergency medicine-trained physicians are proficient in the insertion of transvenous pacemakers. Ann Emerg Med 2004;43:469-74.

(English Language Editor: J. Reynolds) 